\title{
Recurrent Chondroid Syringoma of the Alar Rim
}

\author{
Young Mook Yun, \\ Seungho Shin, \\ Hyunwoo Kyung, \\ Seung Han Song, \\ Nakheon Kang \\ Department of Plastic and Reconstructive \\ Surgery, Chungnam National University \\ School of Medicine, Daejeon, Korea
}

No potential conflict of interest relevant to this article was reported.

\begin{abstract}
Chondroid synringoma (CS), pleomorphic adenoma of skin, is a benign tumor found in the head and neck region. CS was first reported in 1859 by Billorth for the salivary gland tumor. The usual presentation is an slowly growing, asymptomatic mass. A 53-year-old female with a history of chondroid synringoma had presented with multiple firm, nodular masses found in the left nostril area. The lesion had been excised 8 years prior and was diagnosed histopathologically, but had gradually recurred. Excision of the mass located in subcutaneous layer revealed four whitish, firm tumors surrounded with capsular tissue. Neither recurrence nor complications occurred during the 18 months follow-up period. In the head and neck region, chondroid syringoma should always be considered in differential diagnosis of soft tissue masses despite its rare incidence. For that reason, excisional biopsy with clear margin is the optimal diagnostic as well as therapeutic choice. We report a case of recurred chondroid syringoma on the nose in female patient.
\end{abstract}

Keywords: Pleomorphic adenoma / Skin neoplasm / Recurrence

\section{INTRODUCTION}

Chondroid syringoma, pleomorphic adenoma of skin, is a relatively rare benign skin appendageal tumor that originates from the apocrine sweat gland and is composed of epithelial and mesenchymal tissues [1,2]. Incidence of primary chondroid syringoma are low at less than $0.1 \%$ in most reports [2,3]. Most cases of chondroid syringoma are found in the head and neck region [2]. Uncommonly, it can involve extremities or axillary region, trunk, scalp, genital area [4]. These benign tumors are more frequent in middle-aged or older male patients than in female patients $[1,2]$.

Clinically, chondroid syringomas are slow-growing, painless, and firm. Usually, they are located in the subcutaneous or intracutaneous layer. Because of their silent clinical presentation and rare incidence, differentiation diagnosis of chondroid syringoma is

\section{Correspondence: Nakheon Kang}

Department of Plastic and Reconstructive Surgery, Chungnam National University Hospital, 282 Munhwa-ro, Jung-gu, Daejeon 35015, Korea

E-mail: nhk488@cnu.ac.kr

Received May 23, 2015 / Revised October 14, 2015 / Accepted December 3, 2015 relatively difficult. In most cases, the diagnosis is made only after biopsy and histologic exam. We report here a case with histologically-proven, recurrent chondorid syringoma of the nose and review the literature on recurrence of this tumor.

\section{CASE REPORT}

A 53-year-old female patient presented to the plastic surgery clinic with a recurrent mass in the anterior aspect of left nostril. Eight years prior to presentation, she had the initial mass excised at another hospital and, four years prior, subsequently underwent a repeat excision at our clinic (Fig. 1). At the time, histologic examination of the specimen revealed the lesion to be a chondroid syringoma (Fig. 2). The most recent mass had grown slowly over the period of about 6 months.

Physical examination revealed a firm and painless mass covered by normal mucosa. This $0.5-\mathrm{cm}$ diameter mass was palpable at the anterior aspect of left alar rim (Fig. 3). Excisional biopsy was performed under local anesthesia. The mass was excised via an elliptical incision on the inner mucosal layer. Dissection revealed 

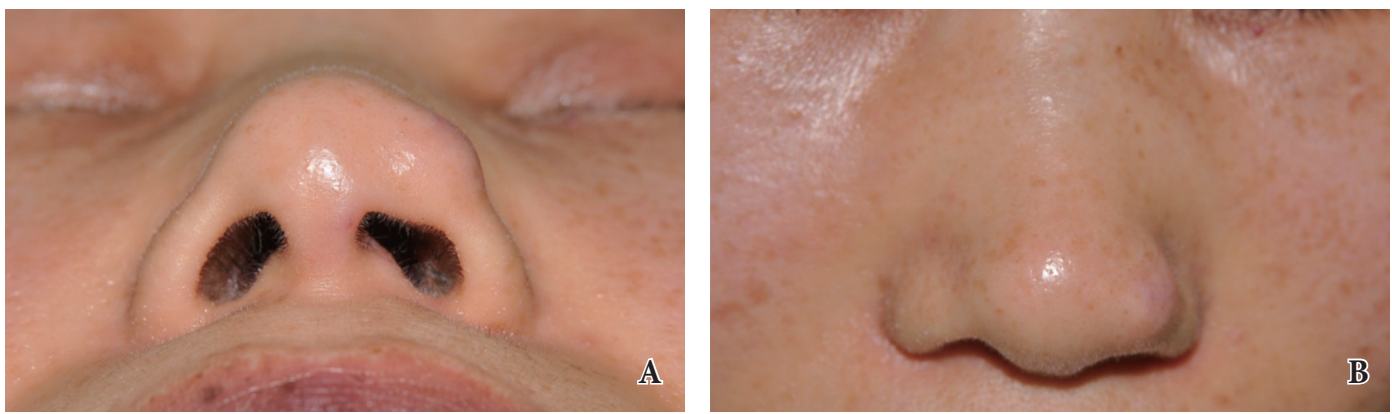

Fig. 1. (A, B) Clinical photographs from 4 years prior. The patient had already undergone excision 8 years prior to presentation and experienced recurrence. A fixed subcutaneous lesion was found in the anterior and posterior aspects of the left alar rim.
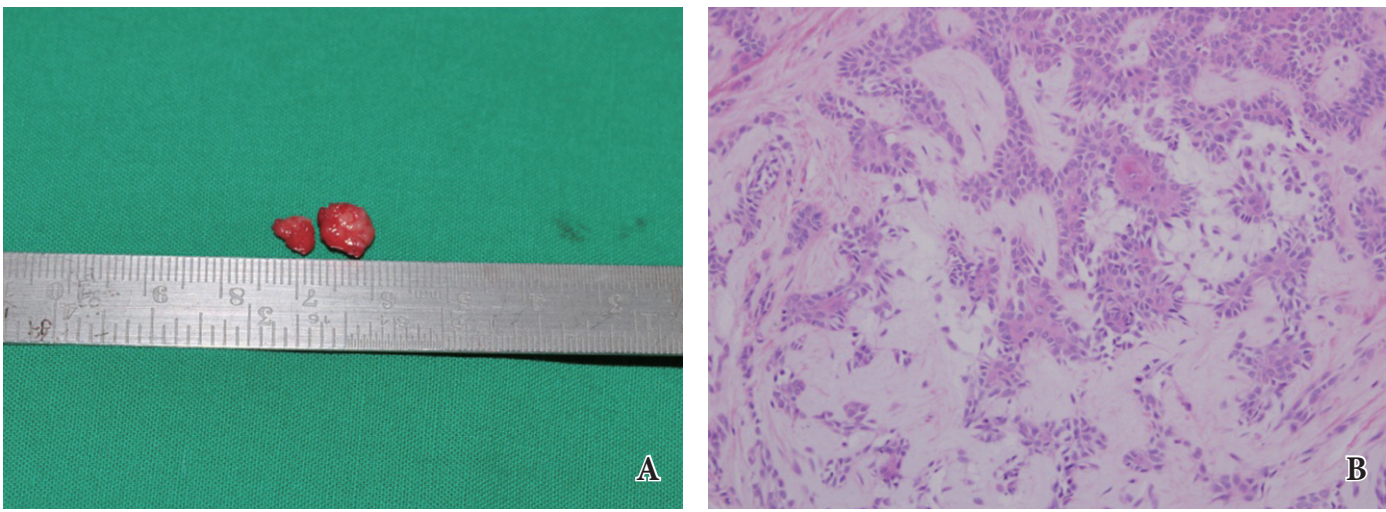

Fig. 2. Intraoperative specimen and histology, 4 years prior to most recent presentation. (A) Gross examination of the surgical specimen demonstrated solid encapsulated masses. (B) Histological examination of the specimen demonstrated proliferative structures of cord and duct in the chondromyxoid stroma. The slide showed a homogeneous basophilic chondroid staining and consisted of mixed epithelial and mesenchymal elements, with epithelial cells arranged in cords and forming tubules with a myoepithelial layer $(\mathrm{H} \& \mathrm{E}, \times 250)$.
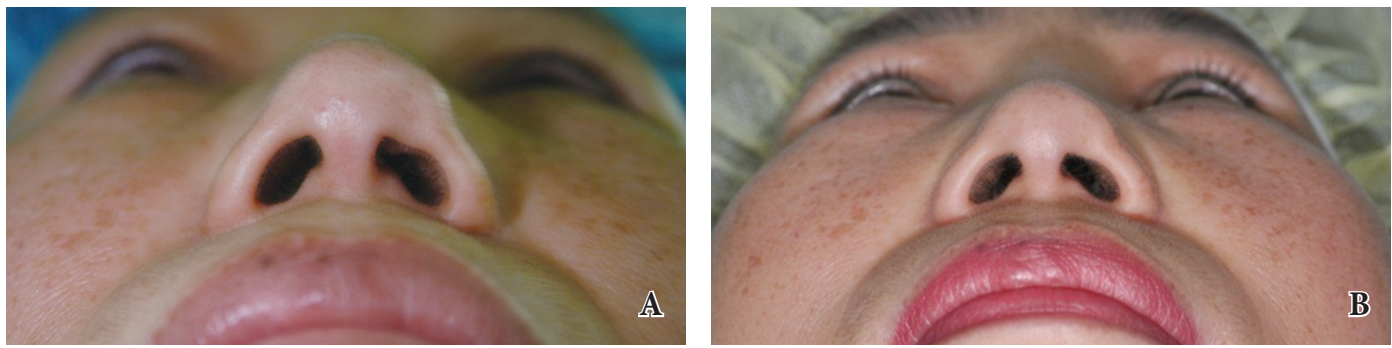

Fig. 3. Most recent preoperative and postoperative photographs. (A) The recurrent mass was firm and painless to palpation and measured $0.5 \mathrm{~cm}$ in diameter. (B) Postoperative photograph at 6 months after surgery. The contour of nasal lining was stable. The wound healed without complications, and no recurrence had been presented during 18-month follow-up.

four individual masses, all of which were located within the subcutaneous layer of outer alar rim and adherent to the underlying cartilage. A thin layer of fibrous tissue had capsulated the mass, which was excised simultaneously from surround tissue. Grossly, the masses were whitish, solid, and lobulated, and varied in size from $0.2 \times 0.1$ to $0.5 \times 0.4 \mathrm{~cm}$.

Histopathologic examination revealed glandular and tubular structures lined by layers of cuboidal epithelial cells and a fibrous or chondroid stroma. The tissue contained mixed epithelial and mesenchymal elements, with epithelial cells arranged in cords and forming tubules with a myoepithelial layer, set in a myxoid or chondroid stroma (Fig. 4). The wound healed without complications, and the patient is without recurrence at 18 months followup period. 

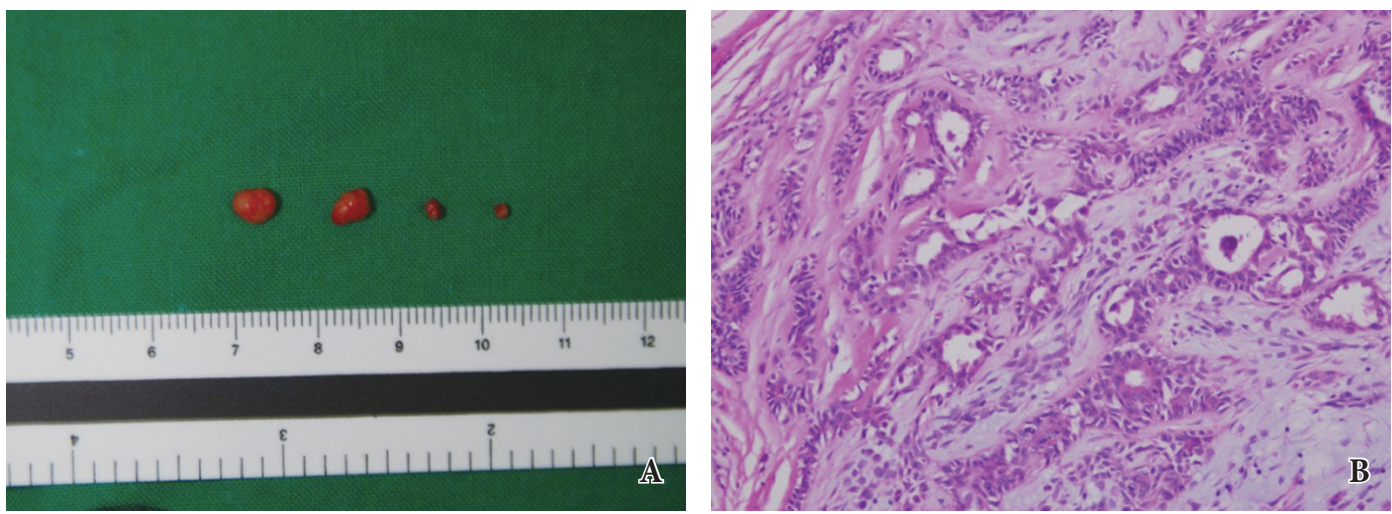

Fig. 4. Intraoperative specimen and histology, most recent. (A) Four, well-circumscribed whitish nodules were excised. Masses varied in size from $0.2 \times 0.1$ to $0.5 \times 0.4 \mathrm{~cm}$. (B) Histopathological examination concurred with previous findings and confirmed the diagnosis of recurrent chondroid syringoma $(\mathrm{H} \& \mathrm{E}, \times 250)$.

\section{DISCUSSION}

Chondroid syringoma is a slow-growing, firm, painless, and wellcircumscribed subcutaneous or intracutaneous nodule. These benign tumors can vary in size, from 0.5 to $3.0 \mathrm{~cm}$ [4]. Due to the nonspecific clinical appearance, the diagnosis of chondroid syringoma can only be made after a histopathologic study.

Chondroid syringoma was first reported by Billorth in 1859 for the salivary gland tumor that contained varying amounts of mucoid and cartilaginous components [2]. Virchow and Minssen described these as mixed tumors composed of both epithelial and mesenchymal origins. The name "chondroid syringoma" became widespread because of the sweat gland elements located within a cartilaginous stroma [5-7], which was the histologic finding in our patient from both excisional biopsy specimens (Fig. 4).

Rarely, this tumor differentiates into other various skin adnexal structures, such as hair follicle, apocrine, sebaceous glands. Because of the silent clinical features, chondroid syringoma is often confused with other types of skin tumors, such as dermoid cyst, sebaceous cyst, neurofibroma, basal cell carcinomas, and pilomatricomas $[1,4]$. Local recurrence rates of chondroid syringoma is not precisely established. In most cases, recurrence occurs as a result of incomplete excision. Routine follow-up is recommended as recurrence rates have been reported from $2.4 \%$ to $10 \%$ [8]. In series of 33 cases of benign or low-grade cytology, Hornick and Fletcher [9] reported that 6 patients (18\%) experienced local recurrence af- ter total excision. The authors noted that no clinical or histologic features were correlated with local recurrence.

Many treatment options have been suggested for chondroid syringoma. However, the treatment of choice is total surgical excision with negative margins because of the possibility of malignant transformation $[1,2,6]$. As with any excision of facial tumor, aesthetic and functional structures need to be preserved as much as possible. With regular follow up, recurrent lesions can be treated by re-excision. Although most chondroid syringoma has benign characteristics, several cases of malignant changes have been reported $[6,9]$. On pathologic exam, the specimen should be evaluated closely to confirm the diagnosis and to rule out malignancy. The malignant form of chondroid syringoma occurs predominantly in women, and is found more commonly on the extremities $[3,6]$. Tumors greater than $3 \mathrm{~cm}$ in size, or with rapid growth, are more likely to conceal malignancy. For malignant chondroid syringoma, the treatment of choice is wide excision and adjuvant radiation therapy with or without chemotherapy [10]. Although chondroid syringoma is not a common disease, surgeons are often the first to interview patients with a mass of the head and neck region. In middle-aged patients, chondroid syringoma should always be kept in mind when approaching a newly growing or recurrent soft tissue mass, as this may be easily treated by simple surgical excision. Accurate history taking and precise histopathologic examination is essential for the diagnosis of chondroid syringoma. Our case emphasizes the importance of 
complete excision without destroying important aesthetic and functional units.

\section{REFERENCES}

1. Agrawal A, Kumar A, Sinha AK, Kumar B, Sabira KC. Chondroid syringoma. Singapore Med J 2008;49:e33-4.

2. Mebazaa A, Trabelsi S, Denguezli M, Sriha B, Belajouza C, Nouira R. Chondroid syringoma of the arm: an unusual localization. Dermatol Online J 2006;12:14.

3. Kerimoglu U, Aydingoz U, Ozkaya O, Aksu AE, Ergen FB. MRI of a benign chondroid syringoma. Br J Radiol 2006;79:e59-61.

4. Yavuzer R, Basterzi Y, Sari A, Bir F, Sezer C. Chondroid syringoma: a diagnosis more frequent than expected. Dermatol Surg 2003;29:179-81.
5. Park CY, Chi JY, Suh YL, Kim YD. Chondroid syringoma of the eyelid. J Korean Ophthalmol Soc 2003;44:1684-8.

6. Sivamani R, Wadhera A, Craig E. Chondroid syringoma: case report and review of the literature. Dermatol Online J 2006;12:8.

7. Hirsch P, Helwig EB. Chondroid syringoma. Mixed tumor of skin, salivary gland type. Arch Dermatol 1961;84:835-47.

8. Ceylan A, Celenk F, Poyraz A, Uslu S. Pleomorphic adenoma of the nasal columella. Pathol Res Pract 2008;204:273-6.

9. Hornick JL, Fletcher CD. Myoepithelial tumors of soft tissue: a clinicopathologic and immunohistochemical study of 101 cases with evaluation of prognostic parameters. Am J Surg Pathol 2003;27:1183-96.

10. Barnett MD, Wallack MK, Zuretti A, Mesia L, Emery RS, Berson AM. Recurrent malignant chondroid syringoma of the foot: a case report and review of the literature. Am J Clin Oncol 2000;23:227-32. 